\title{
Lines of Zahn in the Splenic Vein
}

\author{
Armin Rashidi ${ }^{1}$ Scott Gilles ${ }^{2}$ Michael A. Linden ${ }^{2}$ \\ ${ }^{1}$ Division of Hematology, Oncology, and Transplantation, \\ Department of Medicine, University of Minnesota, Minneapolis, \\ Minnesota, United States \\ ${ }^{2}$ Division of Hematopathology, Department of Laboratory Medicine \\ and Pathology, University of Minnesota, Minneapolis, Minnesota, \\ United States
}

Thromb Haemost 2018;118:957-958.

Two years after a reduced-intensity umbilical cord blood haematopoietic cell transplantation for Philadelphia chromosome-positive B cell acute lymphoblastic leukaemia, a 62 -year-old man presented with acute-onset right upper quadrant pain. Four weeks before this presentation, he had an episode of acute pancreatitis associated with disseminated zoster infection. This was demonstrated on an otherwise unremarkable, contrast-enhanced, abdominal computed tomography (CT) scan. A repeat abdominal CT scan at the time of the most recent presentation revealed an enlarged, diffusely hypodense spleen (suggestive of complete infarction) with lobulated contour and a non-occlusive distal splenic vein thrombus (-Fig. 1A, arrow), new from the prior study. There was no radiographic evidence of
Address for correspondence Armin Rashidi, MD, PhD, Division of Hematology, Oncology, and Transplantation, Department of Medicine, University of Minnesota, 14-132 PWB, 516 Delaware Street SE, Minneapolis, MN 55455, United States (e-mail: arashidi@umn.edu). pancreatitis on this imaging. Positron emission tomography scan was confirmatory, showing no uptake by the spleen (-Fig. 1B). The patient underwent open splenectomy during which multiple adhesions in the peripancreatic/perisplenic area were found. Pathological examination showed an entirely infarcted spleen and thrombosed splenic vein showing lines of Zahn (-Fig. 2A, B). Venous location of the thrombus was confirmed also by an elastin stain (Verhoeff-Van Gieson), staining the adjacent artery but not the vein (-Fig. 2C, arrow). Extensive laboratory work up for malignancy, infection, autoimmunity and graft-versus-host disease was negative. A diagnosis of splenic infarction due to splenic vein thrombosis related to recent pancreatitis was established.

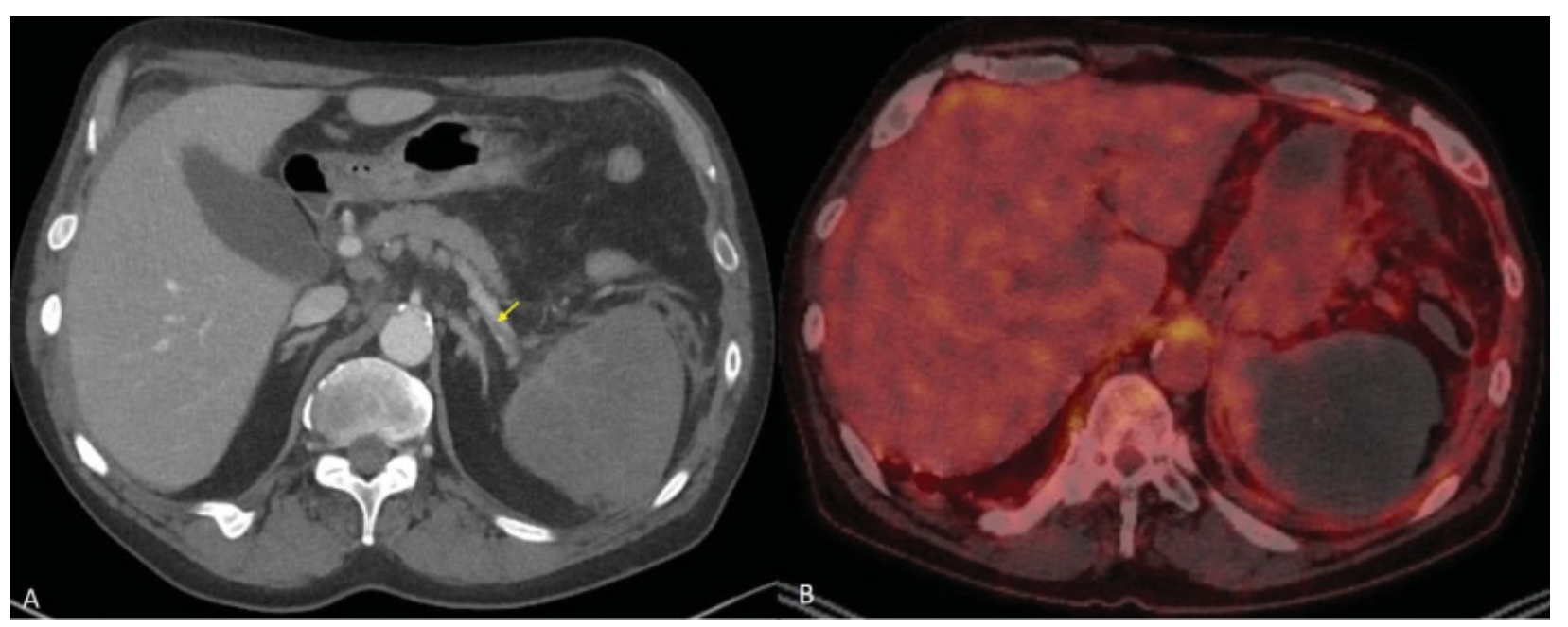

Fig. 1 Splenic vein thrombosis and splenic infarct. (A) Splenic vein thrombosis (arrow) and a hypodense spleen on computed tomography (CT) scan. (B) No splenic uptake on positron emission tomography (PET) scan.

received

January 22, 2018

accepted after revision

March 14, 2018
Copyright @ 2018 Schattauer

DOI https://doi.org/

$10.1055 / \mathrm{s}-0038-1642610$.

ISSN 0340-6245. 


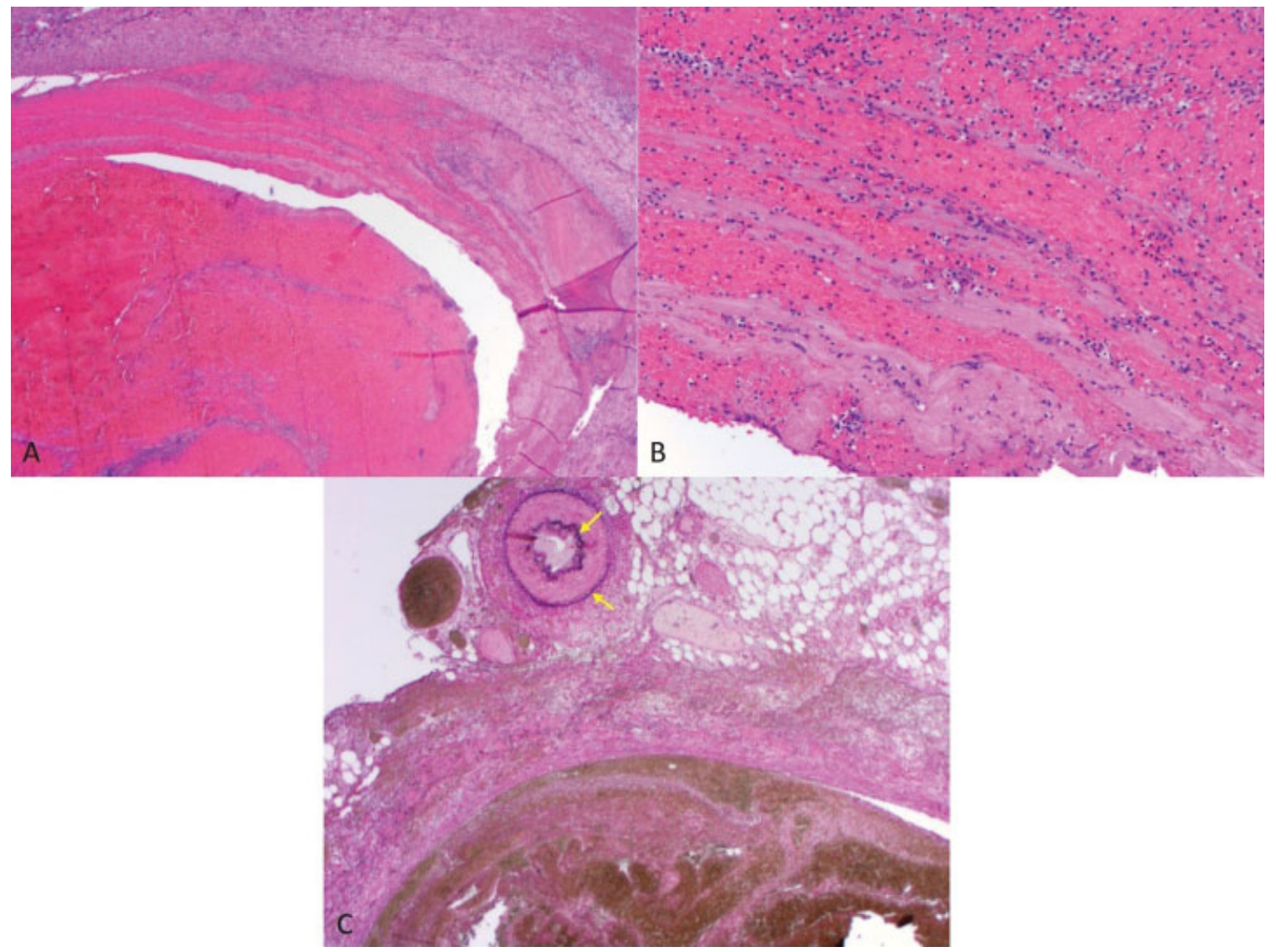

Fig. 2 Splenic vein thrombosis. (A) Splenic vein thrombosis. (B) Lines of Zahn. (C) Elastin stain (Verhoeff-Van Gieson; arrows) positive in the adjacent artery but not the vein.

Lines of Zahn are characteristic of thrombi formed at the sites of rapid arterial blood flow. Successive deposition of platelets and fibrin (pink layers) alternating with red cells (red layers) indicate clot formation in flowing blood and create laminations that define lines of Zahn, often used to distinguish ante-mortem thrombi from post-mortem clots. Formation of lines of Zahn in a typically low-flow vein is unusual. Intense local inflammation due to pancreatitis in our patient may have induced rapid blood flow in the splenic vein and promoted the formation of lines of Zahn.
Funding
None.
Conflict of Interest
None. 